\title{
Contribuciones a la generalización del problema de compensación por grupos de Helmert-Pranis Pranievich
}

\author{
IOAN POPESCU
}

\begin{abstract}
The paper presents in a generalized form the problem of the geodetic network adjustment by the Helmert-Pranis Pranievich groups method (groups with junction points included or not).

The adjustment problem, as well as the cofactor matrix derivation for the partialindependent and linkage unknowns, was completely formulated by transformed weight matrix delinition and usage.

A complete sequence of the computing stages for the geodetic networks divided into groups without junction points was given for eficient programming of adjustment processing on computer.

A practical example illustrates the identity of the solutions and the cofactors obtained by the group adjustment proposed to those obtained by the block adjustment.
\end{abstract}

\section{INTRODUCCION}

La aparición de los métodos de compensación por grupos fue impuesta por las necesidades prácticas de solución rigurosa de grandes redes geodésicas. El objeto concreto de emplear estos procedimientos -obtener eficiencia en los cálculos de compensación-fue realizado en el pasado por la utilización simultánea de varios especialistas en los principales cálculos de compensación de los grupos separados de la red bloque.

El crecimiento de las posibilidades de cálculo empleando computadores electrónicos no ha disminuido la importancia de estos procedimientos. Seguramente que, en principio, ya no se puede hablar más de las modalidades de resolver propuestas antes de la elaboración de éstas, sino que es necesario encontrar otras vías de solución, en conjunción directa con la utilización eficiente de los computadores.

1980 Mathematics Subject Classification (1985 revision): 35F05, 35F20.

Editorial de la Universidad Complutense. Madrid, 1988. 
Por otro lado, el desarrollo de la teoria de errores de observación, especialmente el cálculo de los cofactores (Tienstra, 1956; Linnik, 1960; Linkwitz, 1960) y de la teoría de matrices generalizadas (Bjerhammar, 1973), han ofrecido la posibilidad de resolver por completo los problemas de compensación, incluyendo la estimación de la precisión de las incógnitas, de las observaciones compensadas y de funciones de éstas, así como la generalización de ellas.

Todo esto ha hecho necesaria y también posible la reconsideración del problema de compensación por grupos de las redes grandes.

Este articulo pone en evidencia algunos aspectos de esta problemática, considerando conocido el modelo clásico de ajuste (Pranis-Pranievici, 1956; Lörinczi, 1964; Wolf, 1968; Ghitau, 1970; Dragomir et al., 1982).

\section{DESARROLLO GENERAL DEL PROBLEMA DE COMPENSACION POR EL METODO DE OBSERVACIONES INDIRECTAS}

\subsection{El problema}

Consideremos la red geodésica grande de la figura 1 (anexo A) que fue dividida en «n» grupos.

\section{Observación}

El número de grupos se puede elegir sin restricciones pero, desde el punto de vista práctico, la división en grupos tiene que ser realizada de manera que asegure la introducción en cada uno del número óptimo de puntos geodésicos. Tenemos que entender esto en conexión directa con las necesidades prácticas impuestas y con las posibilidades de un computador (o de un programa) de invertir una matriz de dimensiones antes establecidas. Si se tiene en cuenta esta recomendación, el número total de puntos de unión entre grupos $\left(n_{L}\right)$ no podrá exceder el número máximo de puntos que entran en un grupo separado $\left(n_{\max }\right)$, esto es:

$$
n_{L} \leqslant n_{i \max }
$$

Nos proponemos, empleando los principios de la división en grupos de las redes geodésicas, resolver en el caso general el problema de compensación de Helmert-Pranis Pranievich con el método de observaciones indirectas ponderadas. 
Para cualquier grupo (ii), el sistema de ecuaciones de observación se puede presentar en la forma:

$$
V_{i}=B_{i} X_{i}+\sum_{\substack{j=1 \\ j \neq i}}^{n} C_{i j} X_{i j}+L_{i}
$$

con la matriz de pesos $P_{i}(i=1,2, \ldots, n)$.

Los vectores (Vector $=$ matriz con una dimensión) y las matrices de la relación (1.2) tienen la significación presentada en anexo B (B1).

Empleando las matrices definidas en el anexo B (B2) el sistema general de ecuaciones de observación para todos los «n» grupos, puede ser escrito de la forma (Mihailovič, 1978):

$$
V=B X+C X_{L}+L
$$

o poner la suma de los dos primeros términos del segundo miembro en forma de un producto de matrices,

$$
V=\left[\begin{array}{ll}
B & C
\end{array}\right] \cdot\left[\begin{array}{c}
X \\
X_{L}
\end{array}\right]+L
$$

con la matriz de pesos $P$.

1.2. Generalización del problema de compensación.

Definición de la matriz de pesos transformada

La solución del sistema general de ecuaciones de observación (1.3), con la condición de mínimo,

$$
V^{T} P V=\text { minim }
$$

tiene el siguiente desarrollo:

a) El sistema general de ecuaciones normales es

$$
\begin{aligned}
& B^{T} P B X+B^{T} P C X_{L}+B^{T} P L=0 \\
& C^{T} P B X+C^{T} P C X_{L}+C^{T} P L=0
\end{aligned}
$$

b) El vector de las incógnitas de unión, $X_{L}$, se obtiene resolviendo el sistema (1.5). De la primera ecuación se obtiene directamente

$$
X=-\left(B^{T} P B\right)^{-1}\left(B^{T} P C X_{L}+B^{T} P L\right)
$$


Sustituyendo el valor de $X$ obtenido con (1.6) en la segunda ecuación, resulta:

$$
C^{T}\left\{P-P B\left(B^{T} P B\right)^{-1} B^{T} P\right\} C X_{L}+C^{T}\left\{P-P B\left(B^{T} P B\right)^{-1} B^{T} P\right\} L=0
$$

Podemos definir la matriz:

$$
\begin{gathered}
P-P B\left(B^{T} P B\right)^{-1} B^{T} P=P\left\{E-B\left(B^{T} P B\right)^{-1} B^{T} P\right\}= \\
=P\left\{E-P B\left(B^{T} P B\right)^{-1} B^{T}\right\}=P
\end{gathered}
$$

como la matriz de pesos transformada, donde $E$ representa la matriz unidad (compuesta) correspondiente.

Por la definición de la matriz de pesos transformada (1.8), el vector general de todas las incógnitas de unión, $X_{L}$, resulta de $(1,7)$, en la forma:

$$
X_{L}=-\left(C^{T} \bar{P} C\right)^{-1} C^{T} \bar{P} L
$$

c) La matriz general cofactor de las incógnitas de unión se obtiene aplicando la ley de propagación de los cofactores (Linkwitz, 1960), teniendo en cuenta que la matriz cofactor de las observaciones es la inversa de la matriz de pesos $\left(Q=P^{-1}\right)$, resulta:

$$
Q_{x_{L}}=\left(C^{T} \bar{P} C\right)^{-1} C^{\gamma} \bar{P} P^{-1} \bar{P} C\left(C^{\gamma} \bar{P} C\right)^{-1}
$$

Puesto que los productos matriciales $\bar{P}_{i .} \cdot P_{i}^{-1}=P_{i}^{-1} \cdot \bar{P}_{i}=\bar{P}_{i 0}$, representan una "matriz unidad especial»" con el significado de la denominación establecida por Bjerhammar (Linkwitz, 1960; Bjerhammar, 1973) se puede demostrar la identidad $\bar{P}_{i} \cdot P_{i} \cdot \bar{P}_{i}=\bar{P}_{i}$ (relación E1-anexo E). Teniendo en cuenta esta identidad y la estructura de las matrices $P^{-1}$ y $\bar{P}$, resulta:

$$
Q_{X_{L}}=\left(C^{T} \bar{P} C\right)^{-1} C^{T} \bar{P} C\left(C^{T} \bar{P} C\right)^{-1}
$$

o, en definitiva,

$$
Q_{x_{L}}=\left(C^{T} \bar{P} C\right)^{-1}
$$

d) El vector general de las incógnitas parcial-independientes se obtiene con (1.6), teniendo en cuenta (1.9), en la forma

$$
X=-\left(B^{T} P B\right)^{-1} B^{T} P L+\left(B^{T} P B\right)^{-1} B^{T} P C\left(C^{T} \bar{P} C\right)^{-1} C^{T} \bar{P} L
$$

e) La matriz general cofactor de las incógnitas parcial-independientes resulta con (1.12), siguiendo una via similar a la presentada antes (punto $c$ ), 
teniendo en cuenta la expresión (1.8); después de algunas operaciones resulta

$$
Q_{x}=\left(B^{T} P B\right)^{-1}+\left(B^{T} P B\right)^{-1} B^{T} P C\left(C^{T} P C\right)^{-1} C^{T} P B\left(B^{T} P B\right)^{-1}
$$

El proceso de compensación se cierra con la etapa del cálculo de los residuos bajo la relación (1.3), en la cual ahora todos los elementos son conocidos y con la estimación de la precisión empleando la suma de los cuadrados de los residuos estimados ( $\left.V^{T} P V\right)$.

Con la generalización presentada antes fue obtenido, conforme con la relación (1.11), un primer resultado importante de la utilización de la matriz de pesos transformada: en la compensación por grupos de redes geodésicas, los cofactores de las incógnitas de unión se obtienen directamente de la matriz inversa del sistema normal (reunido) de las incógnitas de unión. Este resultado pone otra vez de actualidad el procedimiento de Helmert-Pranis Pranievich.

\section{GENERALIZACION DEL PROBLEMA DE COMPENSACION PARA REDES GEODESICAS DIVIDIDAS EN GRUPOS SIN PUNTOS NUDALES}

\subsection{Consideraciones sobre la división en grupos de las redes geodésicas}

La división general en grupos presentada en la Figura 1 (anexo A) y, respectivamente, la definición de las matrices conforme con las relaciones $(B 2$, anexo B) es convencional, porque desde un punto de vista práctico, cualquier grupo «i» sólo puede estar en relación con algunos grupos más (ordinariamente menos de 10 grupos). Pero también, en esta situación, la matriz $\mathrm{C}$ es bastante complicada y la división de las observaciones (especialmente en las redes de triangulación) en puntos nudales, pone dificultades que llegan hasta la construcción de las ecuaciones de observación (especialmente en vía de automatización). Para eliminar estos inconvenientes, un primer paso se hace proponiendo que las redes geodésicas sean divididas en grupos sin puntos nudales, esto es, que cada grupo tenga no más de dos grupos vecinos. Esta propuesta es materializada esquemático en la figura 2, anexo A. Para observar las ventajas de la propuesta hecha basta comparar las dimensiones de las matrices de los sistemas generales de las ecuaciones de observación de la división en grupos con puntos nudales (relación Al, anexo A) y sin puntos nudales (relación A2, anexo A).

\subsection{El problema de compensación}

La solución del sistema general de ecuaciones de observación (1.3), teniendo las dimensiones disminuidas (conforme con la relación A2, anexo A) 
procede de forma similar al desarrollo presentado en el punto 1.2, con la diferencia de que el vector general de las incógnitas de unión $X_{L}$ y la matriz $C$ tienen el aspecto presentado en el anexo $\mathrm{C}(\mathrm{Cl}$, respectivamente $\mathrm{C} 2$ ).

\subsection{Particularización para la división de las redes en grupos $(n=3)$}

En el caso en que una red geodésica sea dividida en 3 grupos, sin puntos nudales, el sistema de ecuaciones normales resulta en la forma:

$$
\begin{aligned}
& {\left[\begin{array}{ccc}
B_{1}^{T} P_{1} B_{1} & 0 & 0 \\
0 & B_{2}^{T} P_{2} B_{2} & 0 \\
0 & 0 & B_{3}^{T} P_{3} B_{3}
\end{array}\right] \cdot\left[\begin{array}{l}
X_{1} \\
X_{2} \\
X_{3}
\end{array}\right]+} \\
& +\left[\begin{array}{cc}
B_{1}^{T} P_{1} C_{12} & 0 \\
B_{2}^{T} P_{2} C_{21} & B_{2}^{T} P_{2} C_{23} \\
0 & B_{3}^{T} P_{3} C_{32}
\end{array}\right] \cdot\left[\begin{array}{l}
X_{12} \\
X_{23}
\end{array}\right]+\left[\begin{array}{l}
B_{1}^{T} P_{1} L \\
B_{2}^{T} P_{2} L_{2} \\
B_{3}^{T} P_{3} L_{3}
\end{array}\right]=0 \\
& {\left[\begin{array}{ccc}
C_{12}^{T} P_{1} B_{1} & C_{21}^{T} P_{2} B_{2} & 0 \\
0 & C_{23} P_{2} B_{2} & C_{32} P_{3} B_{3}
\end{array}\right] \cdot\left[\begin{array}{c}
X_{1} \\
X_{2} \\
X_{3}
\end{array}\right]+} \\
& +\sqrt{\left[\begin{array}{cc}
C_{12}^{T} P_{1} C_{12}+C_{21}^{T} P_{2} C_{21} & C_{21}^{T} P_{2} C_{23} \\
C_{23}^{T} P_{2} C_{21} & C_{23}^{T} P_{2} C_{23}+C_{32}^{T} P_{3} C_{32}
\end{array}\right] \cdot\left[\begin{array}{l}
X_{12} \\
X_{23}
\end{array}\right]+} \\
& +\left[\begin{array}{c}
C_{12}^{T} P_{1} L_{1}+C_{21}^{T} P_{2} L_{2} \\
C_{23}^{T} P_{2} L_{2}+C_{32}^{T} P_{3} L_{3}
\end{array}\right]=0
\end{aligned}
$$

Después de las sustituciones correspondientes y la introducción de las matrices de pesos transformadas, obtenemos para las incógnitas de unión el siguiente sistema de ecuaciones:

$$
\begin{gathered}
{\left[\begin{array}{cc}
C_{12}^{T} \bar{P}_{1} C_{12}+C_{21}^{T} \bar{P}_{2} C_{21} & C_{21}^{T} \bar{P}_{2} C_{23} \\
C_{23}^{T} \bar{P}_{2} C_{21} & C_{23}^{T} \bar{P}_{2} C_{23}+C_{32}^{T} \bar{P}_{3} C_{32}
\end{array}\right] \cdot\left[\begin{array}{l}
X_{12} \\
X_{23}
\end{array}\right]+} \\
+\left[\begin{array}{c}
C_{12}^{T} \bar{P}_{1} L_{1}+C^{T}{ }_{21} \bar{P}_{2} L_{2} \\
C^{T} \bar{P}_{23} L_{2}+C^{T}{ }_{32} \bar{P}_{3} L_{3}
\end{array}\right]=0
\end{gathered}
$$


Comparando la parte encuadrada del sistema (2.1) con el sistema (2.2) resulta que tienen forma idéntica, diferenciándose el contenido en que los productos matriciales del primer sistema contienen las matrices de pesos iniciales $P_{i}(i=1,2,3)$ y los del segundo sistema, las matrices de pesos transformadas $\bar{P}_{i}(i=1,2,3)$.

La particularización $n=3$ fue hecha con el propósito de distinguir este hecho, que constituye desde el punto de vista práctico otro resultado importante del empleo de los pesos transformados, enseñando que:

i) En el proceso de construcción de los sistemas normạles en grupos basta hacer los productos $B_{i}^{T} P_{i} B_{i}$, siguiéndose que los productos que contienen las matrices $C_{i j}$ serían hechos con los pesos transformados calculados con la relación (1.8), después de calcular las inversas $\left(B_{i}^{T} P_{i} B_{i}\right)^{-1}$ $=N_{i}^{-1}$.

ii) El sistema (2.2) puede ser resuelto de forma independiente.

\subsection{Desarrollo del problema de compensación} con la matriz $C$ definida de nuevo

\subsubsection{La matriz $C$ definida de nuevo}

La matriz $C$ puede ser definida de nuevo como un vector compuesto en el cual los elementos $C_{i}(i=1,2,3, \ldots, n)$ son matrices en relación con todas las incógnitas de unión de la red y tienen la forma presentada en el anexo D (relaciones D1). Desde el punto de vista práctico las matrices $C_{i}$ son matrices con muchos elementos iguales a cero, lo que hace fácil una organización especial de los cálculos matriciales con el computador.

Correspondientemente fueron definidos de nuevo o precisados los otros apuntes generales del anexo B, bajo la forma presentada en el anexo D (relaciones D2-D7).

Con la propuesta de definir de nuevo la matriz $C$ como un vector compuesto se da un segundo paso para aumentar la eficiencia del procedimiento, teniendo en cuenta la programación de los computadores.

\subsubsection{Desarrollo del problema de compensación}

El sistema general de ecuaciones de observación se puede expresar también con la relación (1.3). Teniendo en cuenta la nueva definición de la 
matriz $C$, el sistema de ecuaciones de observación para cualquier grupo «i» se escribe asi:

$$
V_{i}=B_{i} X_{i}+C_{i} X_{L}+L_{i}
$$

con la matriz de pesos $P_{i}(i=1,2, \ldots, n)$.

La solución del problema de compensación tiene el siguiente desarrollo:

a) El sistema general de ecuaciones normales resulta en la forma:

$$
\begin{gathered}
B_{1}^{T} P_{1} B_{1} X_{1}+B_{1}^{T} P_{1} C_{1} X_{L}+B_{1}^{T} P_{1} L_{1}=0 \\
B_{2}^{T} P_{2} B_{2} X_{2}+B_{2}^{T} P_{2} C_{2} X_{L}+B_{2}^{T} P_{2} L_{2}=0 \\
\ldots \ldots \cdots \cdots \cdots \cdots \cdots \cdots \\
\ldots \ldots \cdots+C_{n}^{T} P_{n} B_{n} X_{n}+ \\
B_{n}^{T} P_{n} B_{n} X_{n}+B_{n}^{T} P_{n} C_{n} X_{L}+B_{n}^{T} P_{n} L_{n}=0 \\
\left.C_{1}^{T} P_{1} B_{1} X_{1}+C_{2}^{T} P_{2} B_{2} X_{2}+\cdots+C_{n}^{T} P_{n} C_{n}\right) X_{L}+ \\
+\left(C_{1}^{T} P_{1} C_{1}+C_{2}^{T} P_{2} C_{2}+\cdots+C_{n}^{T} P_{n} L_{n}\right)=0 \\
+\left(C_{1}^{T} P_{1} L_{1}+C_{2}^{T} P_{2} L_{2}+\cdots+\cdots\right.
\end{gathered}
$$

b) Por las sustituciones:

$$
X_{i}=-N_{i}^{-1} B_{i}^{T} P_{i} C_{i} X_{L}-N_{i}^{-1} B_{i}^{T} P_{i} L_{i}
$$

donde $i=1,2, \ldots, n$ y $N_{i}^{-1}=\left(B_{i}^{T} P_{i} B_{i}\right)^{-1}$

e introduciendo las matrices de pesos transformadas:

$$
P_{i}=P_{i}-P_{i} B_{i} N_{i}^{-1} B_{i}^{T} P_{i}
$$

resulta

$$
\begin{aligned}
& \left(\mathrm{C}_{1}^{\mathrm{T}} \bar{P}_{1} C_{1}+C_{2}^{T} \bar{P}_{2} C_{2}+\cdots+C_{n}^{T} \bar{P}_{n} C_{n}\right) X_{L}+ \\
& \left(C_{1}^{T} \bar{P}_{1} L_{1}+C_{2}^{T} \bar{P}_{2} L_{2}+\cdots+C_{n}^{T} P_{n} L_{n}\right)=0
\end{aligned}
$$


El vector de las incógnitas de unión se obtiene directamente de la relación (2.7) en la forma:

$$
X_{L}=-N_{c}^{-1} C^{T} P L
$$

en que

$$
N_{c}^{-1}=\left(C^{\mathrm{T}} \bar{P} C\right)^{-1}
$$

y

$$
C^{T} P C=\sum_{i=1}^{n} C_{i}^{T} P_{i} C_{i} ; \quad C^{T} \bar{P} L=\sum_{i=1}^{n} C_{i}^{T} \bar{P}_{i} L_{i}
$$

c) La matriz cofactor de las incógnitas de unión es, conforme con (1.11),

$$
Q_{X_{L}}=N_{c}^{-1}
$$

d) El vector de las incógnitas parcial-independientes para cualquier grupo «i» es presentado en la expresión (2.5), en la cual $X_{L}$ tiene el valor (2.8), así como por la relación:

$$
X_{i}=X_{i}^{0}-G_{i} X_{L}
$$

donde

$$
G_{i}=N_{i}^{-1} B_{i}^{T} P_{i} C_{i}
$$

y

$$
X_{i}^{0}=-N_{i}^{-1} B_{i}^{T} P_{i} L_{i}
$$

e) La matriz cofactor de las incógnitas parcial-independientes para cualquier grupo «i» resulta en la forma

$$
Q_{X_{i}}=N_{i}^{-1}+N_{i}^{-1} B_{i}^{T} P_{i} C_{i} N_{c}^{-1} C_{i}^{T} P_{i} B_{i} N_{i}^{-1}
$$

o, teniendo en cuenta los apuntes (2.13),

$$
Q_{x_{i}}=N_{i}^{-1}+G_{i} N_{i}^{-1} G_{i}^{T}
$$

f) Suma de los cuadrados de los resicuıos y fórmulas de comprobación. Se pueden probar de forma bastante fácil (anexo E) las siguientes 
fórmulas de comprobación y de cálculo de la suma de los cuadrados de los residuos:

i)

$$
B_{i}^{T} P_{i} V_{i}=0
$$

y respectivamente,

$$
B_{i}^{T} \bar{P}_{i} V_{i}=0
$$

ii)

$$
\sum_{i=1}^{n} C_{i}^{T} P_{i} V_{i}=0
$$

y respectivamente,

$$
\sum_{i=1}^{n} C_{i}^{\mathrm{T}} \overline{\mathrm{P}}_{i} \mathrm{~V}_{i}=0
$$

iii)

$$
\begin{gathered}
\underline{V_{i}^{T} P_{i} V_{i}}=L_{i}^{T} P_{i} L_{i}+L_{i}^{T} P_{i} B_{i} X_{i}+L_{i}^{T} P_{i} C_{i} X_{L}= \\
=L_{i}^{T} \bar{P}_{i} L_{i}+L_{i}^{T} \bar{P}_{i} B_{i} X_{i}+L_{i}^{T} \bar{P}_{i} C_{i} X_{L}=V_{i}^{T} \bar{P}_{i} V_{i} \\
V^{T} P V=V^{T} P V=\sum_{i=1}^{n} V_{i}^{T} P_{i} V_{i}
\end{gathered}
$$

Notamos que, conforme con la relación (2.12), las incógnitas parcialindependientes se obtienen de una suma de dos vectores, en la cual el primero es el vector de las incógnitas, constituido como si fuera un solo grupo «i», y el segundo, como un vector-corrección para el primero, debido a la influencia de las incógnitas de unión. La misma constatación queda válida para la matriz cofactor (2.16) y presenta importancia desde el punto de vista práctico, porque los términos separados pueden ser calculados independientemente. Esta modalidad de desarrolio fue posible gracias a la utilización en la solución del problema de compensación las matrices de pesos transformadas.

Conforme con las relaciones (2.17)-(2.20), para comprobaciones y cálculo del producto $V^{\top} P V$, se pueden emplear tanto las matrices de pesos iniciales $P_{i}$ como las matrices de pesos transformadas $\bar{P}_{i}$.

\section{PROCEDIMIENTO GENERAL DE RESOLUCION Y EJEMPLO NUMERICO}

La organización general de las etapas de cálculo se presenta en el anexo $\mathrm{F}$ (la figura 3). 
Para poner en práctica el procedimiento de compensación por grupos empleando los pesos transformados fue elegida una red de nivelación geométrica, dividida en dos grupos (anexo G) que ha sido compensada antes por varios métodos, incluso con la red bloque (Ghitau, 1970). Los cálculos de compensación están presentados en el anexo G. De la cadena lógica de los cálculos, siguiendo el procedimiento presentado en el anexo $F$, fueron sacadas algunas operaciones, que se pueden rehacer muy fácilmente.

Los resultados obtenidos, respectivamente, de las incógnitas de unión, las incógnitas parcial-independientes y los cofactores de éstas fueron idénticos a los deducidos antes (Ghitau, 1970), lo que prueba, desde el punto de vista práctico, la validez del procedimiento de emplear los pesos transformados en la compensación por grupos de Helmert-Pranis Pranievich de las redes geodésicas, por el método de observaciones indirectas ponderadas.

De la presentación de las etapas de cálculo (anexo F) resultan también las facilidades traidas, con la utilización de las ponderaciones transformadas y los criterios de eficiencia, expuestos en el artículo, a la programación del problema de compensación para un computador electrónico.

ANEXO A.

\section{DIVISION EN GRUPOS DE LAS REDES GEODESICAS}

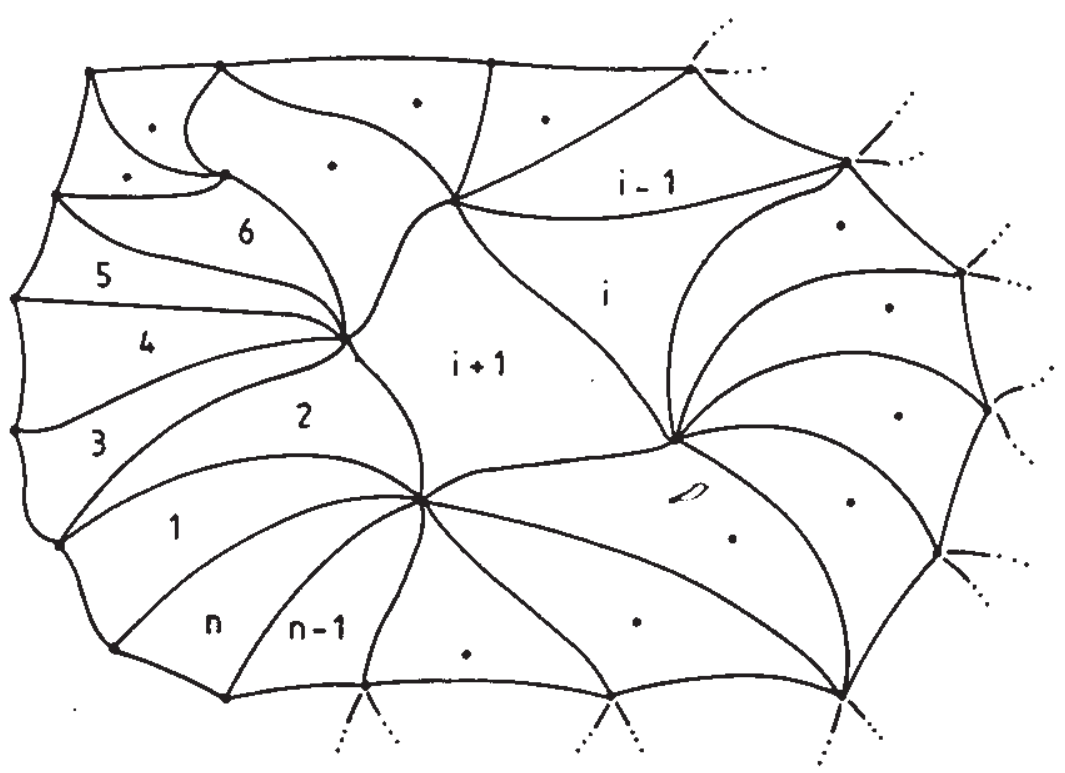

Fig. 1. División general en grupos (con puntos nudales)

$$
V_{n, 1}=B_{n, n} \cdot X_{n, 1}+C_{n, n(n-1) / 2} \cdot X_{L_{n(n-1) / 2,1}}+L_{n, 1}
$$




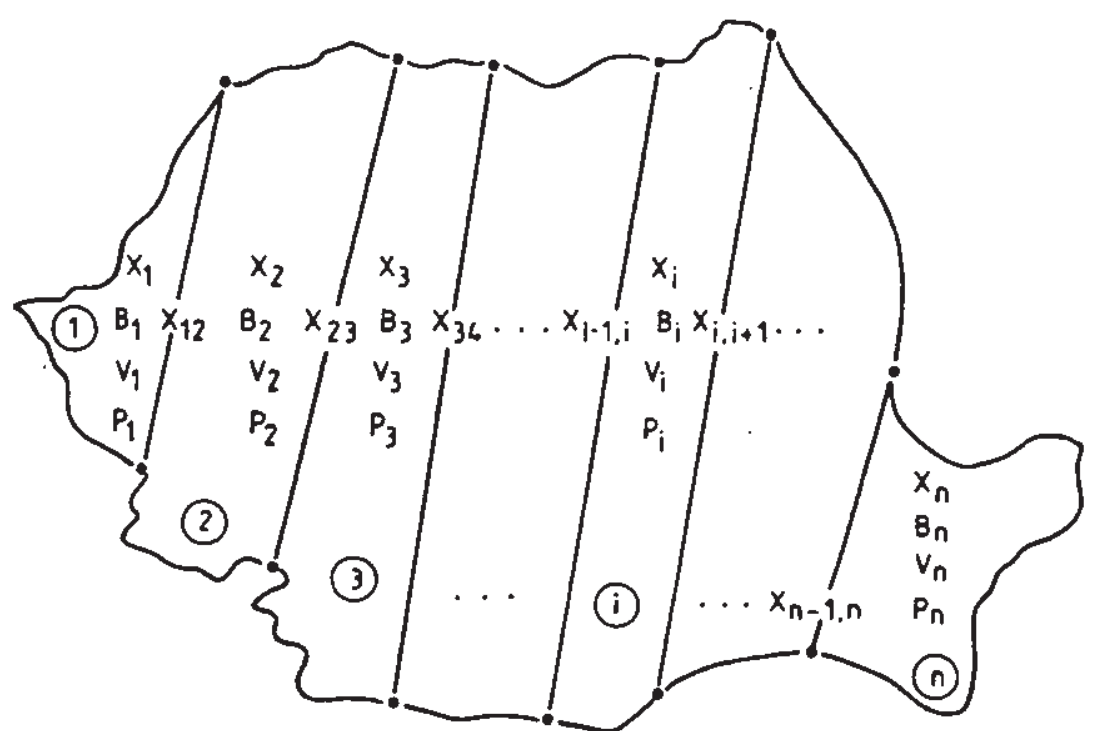

Fig. 2. División en grupos sin puntos nudales

$V_{n, 1}=B_{n, n} \cdot X_{n, 1}+C_{n, n-1} \cdot X_{L_{n-1,1}}+L_{n, 1}$

\section{ANEXO B B1. APUNTES MATRICIALES INICIALES}

Para cualquier grupo «i» $(i=1,2, \ldots, n)$ el significado de las matrices $\mathrm{y}$ vectores es el siguiente:

$X_{i}=$ vector de incógnitas parcial-independientes;

$X_{i j}=$ vector de incógnitas de unión entre los grupos «i» y «j» $\left(X_{i j}=X_{j i}\right)$;

$B_{i}=$ matriz de coeficientes de las incógnitas $X_{i}$ de las ecuaciones de observación;

$C_{i j}=$ matriz de coeficientes de las incógnitas $X_{i j}$ entre los grupos «ij» y «j» $\left(C_{i j} \neq C_{j i}\right)$;

$V_{i}=$ vector de residuos;

$L_{i}=$ vector de observaciones;

$P_{i}=$ matriz diagonal de pesos de las observaciones (inversa de la matriz cofactor $\left.Q_{i}, P_{i}=Q_{i}^{-1}\right) \cdots$

\section{B2. LA DEFINICION DE LAS MATRICES COMPUESTAS}

$$
B=\left[\begin{array}{lllll}
B_{1} & 0 & 0 & \ldots & 0 \\
0 & B_{2} & 0 & \ldots & 0 \\
0 & 0 & B_{3} & \ldots & 0 \\
\ldots & \ldots \ldots \ldots \ldots \ldots \ldots \ldots \ldots . . . & \ldots
\end{array}\right] ; \quad P=\left[\begin{array}{ccccc}
P_{1} & 0 & 0 & \ldots & 0 \\
0 & P_{2} & 0 & \ldots & 0 \\
0 & 0 & 0 & \ldots & B_{n}
\end{array}\right] ;
$$


Contribuciones a la generalización del problema de compensación...

$$
\begin{aligned}
& V^{T}=\left[V_{1} V_{2} V_{3} \ldots V_{n}\right] ; \quad X^{T}=\left[X_{1} X_{2} X_{3} \ldots X_{n}\right] \\
& L^{T}=\left[L_{1} L_{2} L_{3} \ldots L_{n}\right] ; \quad X_{L}^{T}=\left[X_{12} X_{13} \ldots X_{1 n} X_{23} \ldots X_{2 n} \ldots X_{n-1, n}\right] ; \\
& C=\left[\begin{array}{llllllllllll}
C_{12} & C_{13} & \ldots & C_{1 n} & 0 & \ldots & 0 & 0 & \ldots & 0 & \ldots & 0 \\
C_{21} & 0 & \ldots & 0 & C_{23} & \ldots & C_{2 n} & 0 & \ldots & 0 & \ldots & 0 \\
0 & C_{31} & \ldots & 0 & C_{32} & \ldots & 0 & C_{34} & \ldots & C_{3 n} & \ldots & 0 \\
\hdashline 0 & 0 & \ldots & C_{n 1} & 0 & \ldots & C_{n 2} & 0 & \ldots & C_{n 3} & \ldots & C_{n, n-1}
\end{array}\right]
\end{aligned}
$$

\section{ANEXO C}

C1) Vector de las incógnitas de unión:

$$
X_{L}^{T}=\left[X_{12} X_{23} X_{34} \ldots X_{n+1, n}\right]
$$

C2) Matriz de coeficientes de las incógnitas de unión

$$
C=\left[\begin{array}{lllll}
C_{12} & 0 & 0 & \ldots & 0 \\
C_{21} & C_{23} & 0 & \ldots & 0 \\
0 & C_{32} & C_{34} & \ldots & 0 \\
\hdashline \ldots \ldots & \ldots & \ldots & \ldots & \ldots \ldots \ldots \ldots \\
0 & 0 & 0 & \ldots & C_{n-1, n} \\
0 & 0 & 0 & \ldots & C_{n, n-1}
\end{array}\right]
$$

\section{ANEXO D}

D1) La nueva definición de la matriz $C$ como un vector compuesto

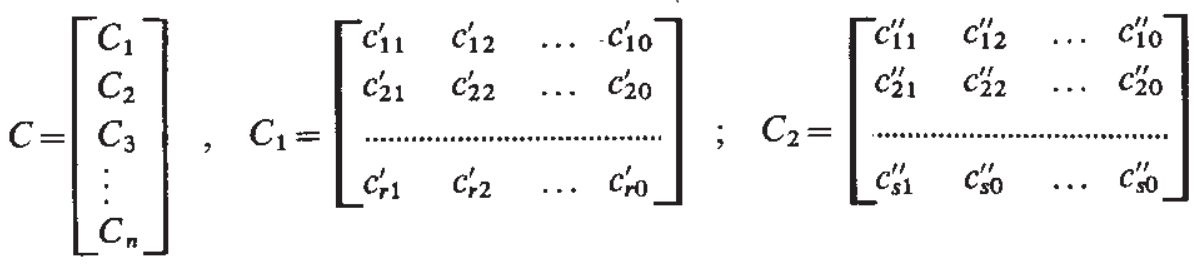

D2) Vector de las incógnitas de unión

$$
X_{L}^{T}=\left[x_{1 L} x_{2 L} \ldots x_{0 L}\right]
$$

D3) Vectores de residuos

$$
V_{1}^{T}=\left[v_{1}^{\prime} v_{2}^{\prime} \ldots v_{r}^{\prime}\right] ; \quad V_{2}^{T}=\left[v_{1}^{\prime \prime} v_{2}^{\prime \prime} \ldots v_{s}^{\prime \prime}\right]
$$


D4) Matrices de coeficientes de las incógnitas parcial-independientes:

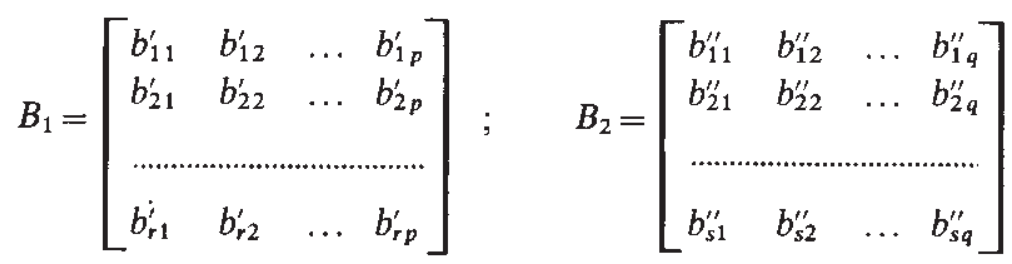

D5) Vectores de las incógnitas parcial-independientes:

$$
X_{1}^{T}=\left[x_{1}^{\prime} x_{2}^{\prime} \ldots x_{p}^{\prime}\right] ; \quad X_{2}^{T}=\left[x_{1}^{\prime \prime} x_{2}^{\prime \prime} \ldots x_{q}^{\prime \prime}\right] ; \ldots
$$

D6) Vectores de observaciones:

$$
L_{1}^{T}=\left[l_{1}^{\prime} l_{2}^{\prime} \ldots l_{r}^{\prime}\right] ; \quad L_{2}^{T}=\left[l_{1}^{\prime \prime} l_{2}^{\prime \prime} \ldots l_{r}^{\prime \prime}\right] ; \ldots
$$

D7) Matrices de pesos:

$$
P_{1}=\left[\begin{array}{llll}
p_{11}^{\prime} & 0 & \ldots & 0 \\
0 & p_{22}^{\prime} & \ldots & 0 \\
\hdashline \ldots \ldots \ldots \ldots \ldots \ldots \ldots & \ldots \ldots . . \\
0 & 0 & \ldots & p_{r r}^{\prime}
\end{array}\right] ; \quad P_{2}=\left[\begin{array}{llll}
p_{11}^{\prime \prime} & 0 & \ldots & 0 \\
0 & p_{22}^{\prime \prime} & \ldots & 0 \\
\ldots \ldots & 0 & \ldots & p_{s s}^{\prime \prime}
\end{array}\right] ; \ldots
$$

\section{ANEXO E}

E1) La identidad $\bar{P}_{i} P_{i}^{-1} \bar{P}_{i}=\bar{P}_{i}$ (relación (1.10)).

Conforme con la definición de la matriz de pesos transformada (para el grupo «i»),

$$
P_{i}=P_{i}-P_{i} B_{i} N_{i}^{-1} B_{i}^{T} P_{i} \text {, donde } N_{i}^{-1}=\left(B_{i}^{T} P_{i} B_{i}\right)^{-1}
$$

tenemos

$$
\bar{P}_{i} P_{i}^{-1} \bar{P}_{i}=\left(P_{i}-P_{i} B_{i} N_{i}^{-1} B_{i}^{T} P_{i}\right)\left(P_{i}^{-1}\right)\left(P_{i}-P_{i} B_{i} N_{i}^{-1} B_{i}^{T} P_{i}\right)
$$

Efectuando los productos matriciales entre los paréntesis, resulta:

$$
\begin{aligned}
P_{i} P_{i}^{-1} \bar{P}_{i} & =P_{i}-P_{i} B_{i} N_{i}^{-1} B_{i}^{T} P_{i}-P_{i} B_{i} N_{i}^{-1} B_{i}^{T} P_{i}+P_{i} B_{i} N_{i}^{-1} B_{i}^{T} P_{i} B_{i} N_{i}^{-1} B_{i}^{T} P_{i} \\
& =P_{i}-P_{i} B_{i} N_{i}^{-1} B_{i}^{T} P_{i} \\
& =\bar{P}_{1} \text { (conforme con la definición); }
\end{aligned}
$$


E2) Comprobaciones y suma de los cuadrados de los residuos $V^{T} P V$ (relaciones $(2.17)-(2.20))$.

a) Empleando las matrices de pesos de las observaciones. «i»):

De las primeras $n$ ecuaciones del sistema (2.4), en la forma (para el grupo

$$
B_{i}^{T} P_{i}\left(B_{i} X_{i}+C_{i} X_{L}+L_{i}\right)=0
$$

resulta enseguida la comprobación $B_{i}^{T} P_{i} V_{i}=0$.

De la misma manera, de la última ecuación del sistema (2.4) se deduce la comprobación $\sum_{i=1}^{n} C_{i}^{T} P_{i} V_{i}=0$ (vector cero). Siguiendo procedimientos similares se deducen las otras identidades en función de las matrices iniciales de pesos de las observaciones $\left(P_{i}\right)$.

b) Empleando las matrices de pesos transformadas.

Para demostrar las identidades en función de las matrices de pesos transformadas se toman en cuenta las relaciones correspondientes deducidas en función de las matrices iniciales de pesos, así como la relación para definir la matriz de pesos transformada, puesta en la forma (para el grupo (ii)):

$$
P_{i}=P_{i}+P_{i} B_{i} N_{i}^{-1} B_{i}^{T} P_{i}
$$

De $B_{i}^{T} P_{i} V_{i}=0$

resulta

$$
B_{i}^{T} P_{i} V_{i}=B_{i}^{T}\left(\bar{P}_{i}+P_{i} B_{i} N_{i}^{-1} B_{i}^{T} P_{i}\right) V_{i}=B_{i}^{T} \bar{P}_{i} V_{i}+B_{i}^{T} P_{i} B_{i} N_{i}^{-1} B_{i}^{T} P_{i} V_{i}
$$

esto es, $B_{i}^{T} \bar{P}_{i} V_{i}=0$.

Multiplicando la relación (E2.2) a la izquierda por $C_{i}^{T}$ y a la derecha por $V_{i}$, tenemos:

$$
C_{i}^{T} P_{i} V_{i}=C_{i}^{T} \bar{P}_{i} V_{i}+C_{i}^{T} P_{i} B_{i} N_{i}^{-1} B_{i}^{T} P_{i} V_{i}
$$

o

$$
C_{i}^{T} P_{i} V_{i}=C_{i}^{T} P_{i} V_{i}
$$

de donde

$$
\sum_{i=1}^{n} C_{i}^{T} P_{i} V_{i}=\sum_{i=1}^{n} C_{i}^{T} P_{i} V_{i}
$$

$$
\text { o } \quad \sum_{i=1}^{n} C_{i}^{T} P_{i} V_{i}=0 \quad \text { porque } \quad \cdot \sum_{i=1}^{n} C_{i}^{T} P_{i} V_{i}=0
$$

Siguiendo procedimientos similares se obtienen también las otras identidades. 


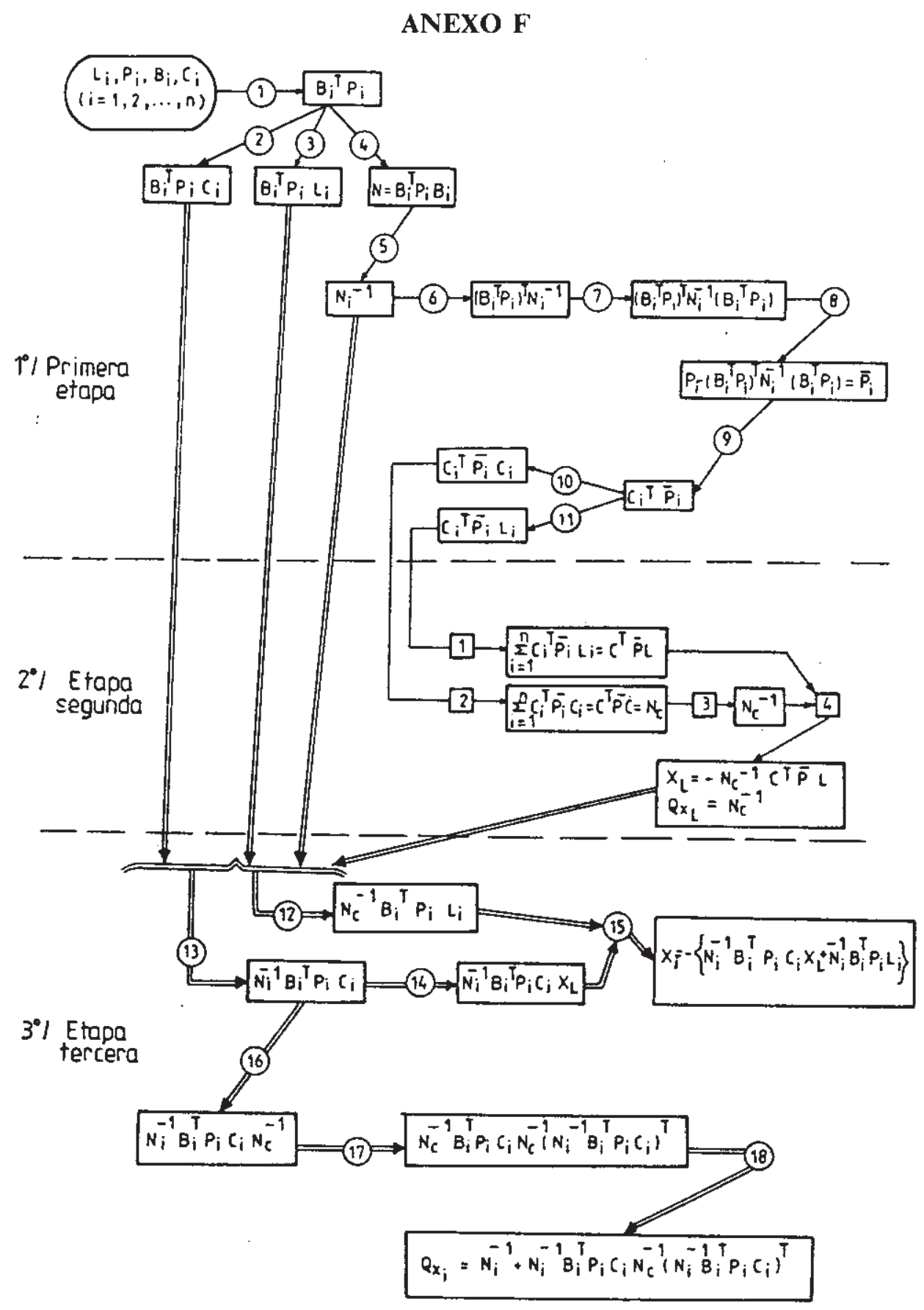

Fig. 3. Organización de las etapas de cálculo. 
ANEXO G

COMPENSACION DE UNA RED DE NIVELACION GEOMETRICA

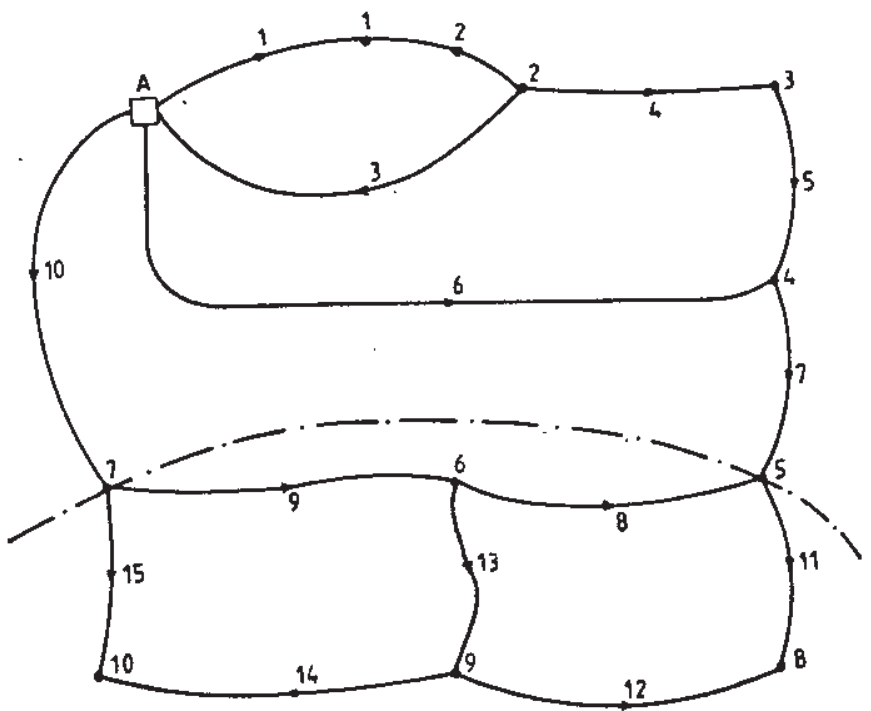

Fig. 4. Bosquejo de la red con la división en grupos.

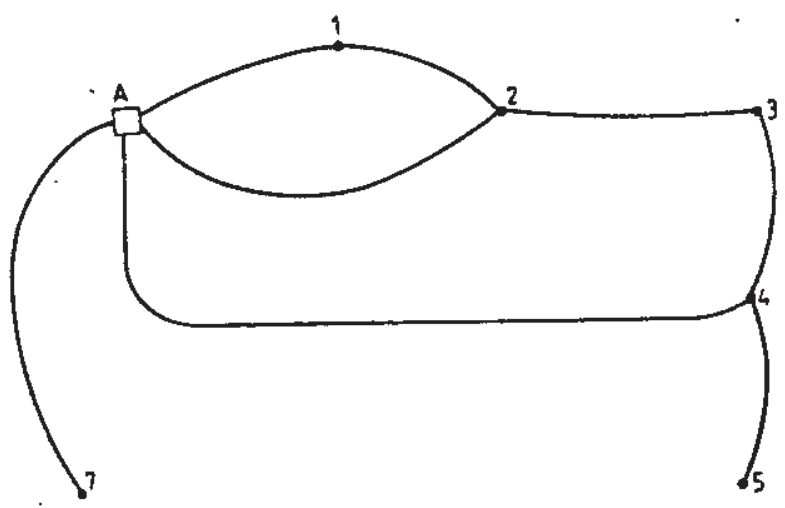

Fig. 5. Grupo número 1.

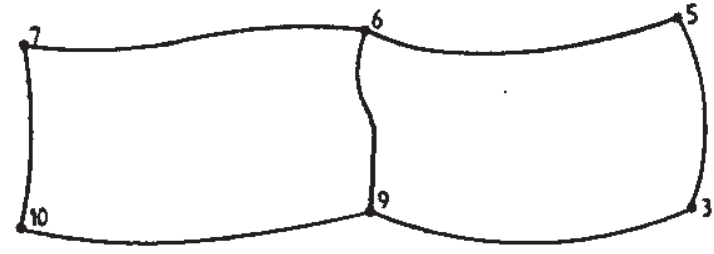

Fig. 6. Grupo número 2. 
TABLA 1

Ecuaciones de observación para el grupo número 1

\begin{tabular}{|c|c|c|c|c|c|c|c|c|c|}
\hline$(\mathrm{mm})$ & $v$ & 1 & 2 & 3 & 4 & 5 & 7 & $\begin{array}{c}1 \\
(m m)\end{array}$ & $p=\frac{10}{S_{K m}}$ \\
\hline$-0,60$ & 1 & 1 & 0 & 0 & 0 & 0 & 0 & 0,0 & 4,0 \\
\hline$-0,59$ & 2 & 1 & -1 & 0 & 0 & 0 & 0 & 0,7 & 4,0 \\
\hline$-0,81$ & 3 & 0 & -1 & 0 & 0 & 0 & 0 & $-1,3$ & 2,0 \\
\hline$-0,19$ & 4 & 0 & -1 & 1 & 0 & 0 & 0 & 0,0 & 4,0 \\
\hline$-0,18$ & 5 & 0 & 0 & -1 & 1 & 0 & 0 & $-2,7$ & 4,3478 \\
\hline 2,44 & 6 & 0 & 0 & 0 & 1 & 0 & 0 & 1,6 & 0,9804 \\
\hline 0,41 & 7 & 0 & 0 & 0 & -1 & 1 & 0 & 0,0 & 4,0 \\
\hline$-2,07$ & 10 & 0 & 0 & 0 & 0 & 0 & 1 & $-0,6$ & 0,7874 \\
\hline & $V_{1}$ & \multicolumn{4}{|c|}{$B_{1}$} & \multicolumn{2}{|c|}{$C_{1}$} & $L_{1}$ & $\operatorname{diag} . P_{1}$ \\
\hline
\end{tabular}

TABLA 2

Ecuaciones de observación para el grupo número 2

\begin{tabular}{|c|c|c|c|c|c|c|c|c|c|}
\hline$(\mathrm{mm})$ & $v$ & 10 & 9 & 8 & 6 & 5 & 7 & $\begin{array}{c}1 \\
(m m)\end{array}$ & $p=\frac{10}{S_{K m}}$ \\
\hline$-0,30$ & 8 & 0 & 0 & 0 & -1 & 1 & 0 & 0,0 & 4,7619 \\
\hline 1,22 & 9 & 0 & 0 & 0 & 1 & 0 & -1 & $-2,8$ & 4,0 \\
\hline 0,05 & 11 & 0 & 0 & 1 & 0 & -1 & 0 & 0,0 & 4,0 \\
\hline$-0,06$ & 12 & 0 & -1 & 1 & 0 & 0 & 0 & 1,1 & 4,0 \\
\hline 1,81 & 13 & 0 & 1 & 0 & -1 & 0 & 0 & 0,9 & 3,4483 \\
\hline 1,87 & 14 & 1 & -1 & 0 & 0 & 0 & 0 & 2,9 & 3,4483 \\
\hline$-1,10$ & 15 & 1 & 0 & 0 & 0 & 0 & -1 & $-5,0$ & 5,8824 \\
\hline & $V_{2}$ & \multicolumn{4}{|c|}{$B_{2}$} & \multicolumn{2}{|c|}{$C$} & $L_{2}$ & $\operatorname{diag} . P_{2}$ \\
\hline
\end{tabular}


Contribuciones a la generalización del problema de compensación...

\section{ETAPA 1 CALCULOS SEPARADOS EN GRUPOS}

$$
\begin{aligned}
& B_{1}^{T} P_{1} B_{1}=\left[\begin{array}{rrcc}
8 & -4 & 0 & 0 \\
-4 & 10 & -4 & 0 \\
0 & -4 & 8,3478 & -4,3478 \\
0 & 0 & -4,3478 & 9,3282
\end{array}\right] ; \quad B_{1}^{T} P_{1} L_{1}=\left[\begin{array}{c}
2,8 \\
-0,2 \\
11,7391 \\
-11,1508
\end{array}\right] \\
& B_{2}^{T} P_{2} B_{2}=\left[\begin{array}{cccc}
9,3307 & -3,4483 & 0 & 0 \\
-3,4483 & 10,8966 & -4 & -3,4483 \\
0 & -4 & 8 & 0 \\
0 & -3,4483 & 0 & 12,2102
\end{array}\right] ; B_{2}^{T} P_{2} L_{2}=\left[\begin{array}{c}
-19,412 \\
-11,2966 \\
4,4 \\
-14,3035
\end{array}\right] \\
& N_{1}^{-1}=\left(B_{1}^{T} P_{1} B_{1}\right)^{-1}=\left[\begin{array}{llll}
0,1707 & 0,0914 & 0,0578 & 0,0270 \\
& 0,1828 & 0,1157 & 0,0539 \\
& & 0,2314 & 0,1079 \\
& & & 0,1575
\end{array}\right] \text {; } \\
& N_{2}^{-1}=\left(B_{2}^{T} P_{2} B_{2}\right)^{-1}=\left[\begin{array}{llll}
0,1277 & 0,0556 & 0,0278 & 0,0157 \\
& 0,1504 & 0,0752 & 0,0425 \\
& & 0,1626 & 0,0212 \\
& & & 0,0939
\end{array}\right]
\end{aligned}
$$

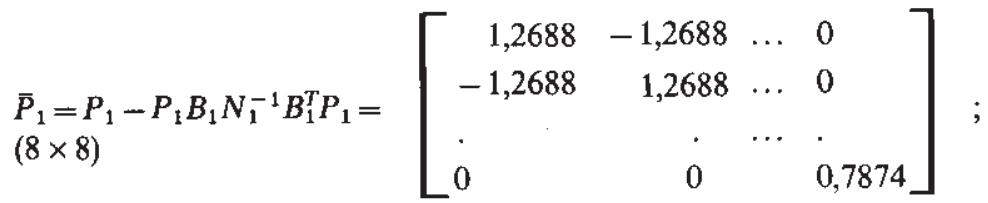

$$
\begin{aligned}
& \begin{array}{l}
\bar{P}_{2}=P_{2}-P_{2} B_{2} N_{2}^{-1} B_{2}^{T} P_{2}=\left[\begin{array}{cccc}
2,6328 & 1,7885 & \ldots & 0,4397 \\
1,7885 & 2,4977 & \ldots & -0,3694 \\
. & . & \ldots & . \\
0,4397 & -0,3694 & \ldots & 1,4631
\end{array}\right] ;
\end{array} \\
& C_{1}^{T} P_{1} C_{1}=\left[\begin{array}{ll}
1,4804 & 0 \\
0 & 0,7874
\end{array}\right] ; \quad C_{1}^{T} \bar{P}_{1} L_{1}=\left[\begin{array}{l}
-1,7012 \\
0,4724
\end{array}\right] ; \\
& C_{2}^{T} \bar{P}_{2} C_{2}=\left[\begin{array}{rr}
3,2220 & -3,2220 \\
-3,3220 & 32220
\end{array}\right] ; \quad C_{2}^{T} P_{2} L_{2}=\left[\begin{array}{r}
-13,5962 \\
13,5962
\end{array}\right] ;
\end{aligned}
$$


ETAPA 2

CALCULOS REUNIDOS PARA $X_{L}$ Y $Q_{X_{L}}$

$$
\begin{aligned}
& C^{T} P C=C_{1}^{T} \bar{P}_{1} C_{1}+C_{1}^{T} \bar{P}_{2} C_{2}=\left[\begin{array}{rr}
4,7024 & -3,2220 \\
-3,2220 & 4,0094
\end{array}\right] \\
& C^{T} \bar{P} L=C_{1}^{T} \bar{P}_{1} L_{1}+C_{2}^{T} P_{2} L_{2}=\left[\begin{array}{r}
-15,2974 \\
13,1238
\end{array}\right] \\
& N_{c}^{-1}=\left(C^{r} P C\right)^{-1}=\left[\begin{array}{ll}
0,4733 & 0,3804 \\
0,3804 & 0,5551
\end{array}\right] \\
& X_{L}=-\left(C^{T} P C\right) C^{T} \bar{P} L=-\left[\begin{array}{r}
-2,2489 \\
1,4660
\end{array}\right] ; \quad Q x_{L}=N_{\mathrm{c}}^{-1} \\
& x_{5}=2,25 \mathrm{~mm} ; x_{7}=-1,47 \mathrm{~mm} ; \quad q_{x 5}=0,47 ; q_{x 7}=0,56
\end{aligned}
$$

ETAPA 3

CALCULOS SEPARADOS EN GRUPOS PARA $X_{i}$ Y $Q_{X_{i}}$

$X_{1}=-N_{1}^{-1} B_{1}^{\top} P_{1} L_{1}-N_{1}^{-1} B_{1}^{\prime} P_{1} C_{1} X_{L}=\left[\begin{array}{r}-0,5927 \\ -0,4912 \\ -0,6825 \\ 1,8416\end{array}\right] \rightarrow \begin{array}{r}x_{1}=-0,60 \mathrm{~mm} ; \\ x_{2}=-0,49 \mathrm{~mm} \\ x_{3}=-0,68 \mathrm{~mm} \\ x_{4}=1,84 \mathrm{~mm} ;\end{array}$

$Q_{X_{1}}=N_{1}^{-1}+N_{1}^{-1} B_{1}^{T} P_{1} C_{1} N_{c}^{-1} C_{1}^{T} P_{1} B_{1} N_{1}^{-1}$

$Q_{X_{1}}=\left[\begin{array}{llll}0,1762 & 0,1024 & 0,0800 & 0,0592 \\ & 0,2048 & 0,1597 & 0,1182 \\ & & 0,3194 & 0,2365 \\ & & & 0,3453\end{array}\right]$;

$q_{x_{1}}=0,18 ; q_{x_{2}}=0,20 ; q_{x_{3}}=0,32 ; q_{x_{4}}=0,35 ;$

$X_{2}=-N_{2}^{-1}{ }_{2}^{T} P_{2} L_{2}-N_{2}^{-1} B_{2}^{T} P_{2} C_{2} X_{L}=\left[\begin{array}{c}2,4341 \\ 3,4579 \\ 2,3034 \\ 2,5448\end{array}\right] \rightarrow \begin{array}{r}x_{10}=2,43 \mathrm{~mm} \\ x_{9}=3,46 \mathrm{~mm} \\ x_{8}=2,30 \mathrm{~mm} \\ x_{6}=2,54 \mathrm{~mm}\end{array}$ 


$$
\begin{aligned}
& Q_{X_{2}}=N_{2}^{-1}+N_{2}^{-1} B_{2}^{T} P_{2} C_{2} N_{c}^{-1} C_{2}^{T} P_{2} B_{2} N_{2}^{-1} \\
& Q_{X_{2}}=\left[\begin{array}{cccc}
0,6271 & 0,5154 & 0,4565 & 0,4718 \\
& 0,5975 & 0,5123 & 0,4884 \\
& & 0,6063 & 0,4591 \\
& & & 0,5389
\end{array}\right] ; \\
& q_{x_{10}}=0,63 ; q_{x_{9}}=0,60 ; q_{x_{0}}=0,61 ; q_{x_{6}}=0,54 .
\end{aligned}
$$

\section{Bibliografia}

[1] BJERHAMMAR, A. Theory of errors and generalized matrix inverses, Elsevier Scientific Pub. Co., Amsterdam, 1973.

[2] Dragomir, V., Ghitau, D., Mihailescu, M., Rotaru, M. Theory of the Earth's Shape, Elsevier Scientific Pub. Co., Amsterdam, 1982.

[3] GHITAU, D., Compensarea si comasarea riguroasa a retelelor geodezice deja compensate, Institutul de Constructii, Bucuresti, 1970.

[4] GHITAU, D. Geodezie si gravimetrie geodezica, Editura Didactică si Pedagogică, Bucuresti, 1984.

[5] LINK WITZ, K. Uber die sistematik verschiedener formen der ausgleichsrechnun, In Z.f.V. nr. 5, 6, 7, 1960.

[6] LINNIK, I. V. Metod naimensih kvadratov i osnovi teorii obrabotki nabludenit, Nedra, Moskva, 1962.

[7] LORINCZI, I. Contributii la teoria compensarii triangulatillor geodezice. Teza de doctorat, Universitatea Bucaresti, Bucaresti, 1964.

[8] Mihallovic, K. Geodezija, Naučna kniga, Beograd, 1978.

[9] Pranis-PraniEVICI, I. J. Rukovodstoo po uravnitelnim vicisleniam triangulatii, Izdatelstvo geodezicesnoi literaturi, Moskva, 1956.

[10] TIENSTRA, J. M., Theory of the adjustement of normaly distributed observations. N. V. Uitgevenij Argus, Amsterdam, 1956.

[11] WoLF, H., Ausgleichungsrechnung nach der methode der kleinsten quadrate, Dümmler's Verlag, Bonn, 1968.

Ministerul Apararii Nationale

Marele Stat Major

Directia Topografica Militara

Bucaresti. Cod. 77302

Republica Socialista Romania 